\title{
In-hospital mortality following treatment with red blood cell transfusion or inotropic therapy during early goal-directed therapy for septic shock: a retrospective propensity-adjusted analysis
}

Dustin G Mark ${ }^{1 *}$, John W Morehouse', Yun-Yi Hung ${ }^{2}$, Mamata V Kene ${ }^{3}$, Andrew R Elms ${ }^{4}$, Vincent Liu², Dustin W Ballard ${ }^{5}$ and David R Vinson ${ }^{6}$

\begin{abstract}
Introduction: We sought to investigate whether treatment of subnormal $(<70 \%)$ central venous oxygen saturation $\left(\mathrm{ScvO}_{2}\right)$ with inotropes or red blood cell (RBC) transfusion during early goal-directed therapy (EGDT) for septic shock is independently associated with in-hospital mortality.

Methods: Retrospective analysis of a prospective EGDT patient database drawn from 21 emergency departments with a single standardized EGDT protocol. Patients were included if, during EGDT, they concomitantly achieved a central venous pressure (CVP) of $\geq 8 \mathrm{~mm} \mathrm{Hg}$ and a mean arterial pressure (MAP) of $\geq 65 \mathrm{~mm} \mathrm{Hg}$ while registering a $\mathrm{ScvO}_{2}<70 \%$. Treatment propensity scores for either RBC transfusion or inotrope administration were separately determined from independent patient sub-cohorts. Propensity-adjusted logistic regression analyses were conducted to test for associations between treatments and in-hospital mortality.

Results: Of 2,595 EGDT patients, 572 (22.0\%) met study inclusion criteria. The overall in-hospital mortality rate was 20.5\%. Inotropes or RBC transfusions were administered for an $\mathrm{ScvO}_{2}<70 \%$ to $51.9 \%$ of patients. Patients were not statistically more likely to achieve an $\mathrm{ScvO}_{2}$ of $\geq 70 \%$ if they were treated with RBC transfusion alone $(29 / 59,49.2 \%, P=0.19)$, inotropic therapy alone $(104 / 226,46.0 \%, P=0.15)$ or both $\mathrm{RBC}$ and inotropic therapy $(7 / 12,58.3 \%, P=0.23)$ as compared to no therapy $(108 / 275,39.3 \%)$. Following adjustment for treatment propensity score, RBC transfusion was associated with a decreased adjusted odds ratio (aOR) of in-hospital mortality among patients with hemoglobin values less than $10 \mathrm{~g} / \mathrm{dL}$ (aOR $0.42,95 \% \mathrm{Cl} 0.18$ to $0.97, P=0.04$ ) while inotropic therapy was not associated with in-hospital mortality among patients with hemoglobin values of $10 \mathrm{~g} / \mathrm{dL}$ or greater (aOR $1.16,95 \% \mathrm{Cl} 0.69$ to $1.96, P=0.57)$.

Conclusions: Among patients with septic shock treated with EGDT in the setting of subnormal $\mathrm{ScVO}_{2}$ values despite meeting CVP and MAP target goals, treatment with RBC transfusion may be independently associated with decreased in-hospital mortality.
\end{abstract}

\footnotetext{
* Correspondence: Dmark28@gmail.com

${ }^{1}$ Department of Emergency Medicine, Kaiser Permanente, 275 West

Macarthur Boulevard, Oakland, CA 94611, USA

Full list of author information is available at the end of the article
} 


\section{Introduction}

Early goal-directed therapy (EGDT), when applied to emergency department (ED) patients with septic shock, can reduce mortality compared to standardized resuscitation that targets central venous pressure (CVP), mean arterial pressure (MAP) and urine output goals [1]. EGDT can be conceptualized as a central venous oxygen saturation $\left(\mathrm{ScvO}_{2}\right)$-guided resuscitation protocol, targeting a goal $\mathrm{ScvO}_{2}$ of $70 \%$ or greater once standardized CVP, MAP and urine output targets have been achieved. $\mathrm{ScvO}_{2}$ values below the $70 \%$ target can be increased with therapies aimed at decreasing oxygen consumption $\left(\mathrm{VO}_{2}\right)$, such as mechanical ventilation, sedation and/or pharmacologically induced paralysis, and with therapies aimed at increasing oxygen delivery $\left(\mathrm{DO}_{2}\right)$, such as volume expansion, inotropic therapy, red blood cell (RBC) transfusion and vasodilator administration [2-5].

Since the landmark EGDT trial [1], impressive advances have been reported in reducing sepsis-related mortality with implementation of EGDT-based resuscitation bundles [6-10]. However, the incremental benefit of the $\mathrm{ScvO}_{2}$-guided $\mathrm{DO}_{2}$ augmentation components of these bundles is unclear [9,11-14]. In particular, the ability of RBC transfusions to augment $\mathrm{DO}_{2}$ in sepsis appears minimal [14-18], and liberal transfusion strategies in high-risk or critically ill patients may result in increased mortality rates [19-23], though observational propensity-matched data has suggested an inverse association between RBC transfusion and mortality among patients hospitalized with sepsis [15,24]. Physiologic studies of inotropic therapy in septic shock have demonstrated heterogeneous individual responses both in terms of global cardiac performance and microcirculatory perfusion, with some studies even suggesting overall harm [13,25-30].

Given these uncertain and variable associations, we specifically examined a subset of patients with septic shock, treated in the ED, with documented eligibility for $\mathrm{DO}_{2}$ augmentation therapies according to an EGDT protocol. Prior research has been limited in this regard since prospective studies of EGDT have reported significantly fewer proportions of patients either eligible for or receiving $\mathrm{DO}_{2}$ augmentation therapies as compared to the landmark EGDT trial [10,31-35]. The specific goals of this investigation were to examine associations between EGDT-recommended $\mathrm{DO}_{2}$ augmentation therapies (RBC transfusion and inotropic therapy) and in-hospital mortality rates using a propensityadjusted analysis. We hypothesized that treatment with $\mathrm{DO}_{2}$ augmentation therapies would be associated with improved in-hospital survival when adjusting for severity of illness and comorbidities.

\section{Material and methods} Study setting and design

We performed a retrospective review of a prospective quality improvement database of ED patients treated for septic shock with EGDT within Kaiser Permanente Northern California (KPNC), an integrated healthcare delivery system, between March 2010 and September 2012. KPNC provides comprehensive care for more than 3.4 million members using an integrated electronic health record (Epic, Verona, WI, USA). Approximately 500 emergency medicine board-certified or board-eligible physicians staff the 21 EDs comprising the KPNC emergency care network. The total annual ED volume for all centers in 2010 was 741,475. Individual ED annual patient census ranged from 17,544 to 65,440 (median 31,726, interquartile range 24,980 to 41,721). All EDs are part of medical centers with adult intensive care units ranging in size from between 8 to 32 beds. Patient-level clinical data is electronically accessible within hierarchical databases as described previously [36].

The study period followed the uniform implementation of the KPNC EGDT protocol (Figures 1 and 2) as part of a system-wide quality improvement initiative focused on the care of patients with sepsis [7]. Implementation included a training program conducted at each facility emphasizing sepsis diagnoses, management and ultrasoundguided thoracic central venous catheterization. Standardized electronic order sets outlining all elements of the EGDT protocol were in place during the study period. All 21 EDs were capable of using a triple lumen central venous catheter to continuously monitor $\mathrm{ScvO}_{2}$ (PreSep Oximetry catheters, Edwards Lifesciences Corporation, Irvine, CA, USA). All RBC products were leukoreduced prior to transfusion. The study was approved by the KPNC Health Services Institutional Review Board (IRB). The IRB waived the requirement for obtaining informed consent from potential study subjects given the minimal risk posed to participants.

\section{Selection of participants}

Electronically recorded data were used to identify patients who simultaneously achieved both a CVP of 8 millimeters mercury $(\mathrm{mm} \mathrm{Hg})$ or greater and a MAP of $65 \mathrm{~mm} \mathrm{Hg}$ or greater (target goals) during the first six hours of eligibility for EGDT. We defined onset of EGDT eligibility in accordance with the 'time zero' definition adopted by the KPNC EGDT treatment algorithm as (1) a resulted peripheral blood lactate level of $4 \mathrm{mmol} / \mathrm{L}$ or greater or (2) a systolic blood pressure measurement less than or equal to $90 \mathrm{~mm} \mathrm{Hg}$ following the administration of $20 \mathrm{ml} / \mathrm{kg}$ of crystalloid fluids over one hour (Figure 1). Time zero was prospectively recorded by clinical quality abstractors over the course of the study period, with documentation often supplemented by the treating clinical team through the use of an EGDT-specific treatment record.

We included patients if they had two or more $\mathrm{ScvO}_{2}$ measurements less than $70 \%$ during the first six hours of EGDT eligibility along with adequate arterial oxygenation $\left(\mathrm{SpO}_{2} \geq 93 \%\right)$ and concomitant (within 15 minutes) $\mathrm{CVP}$ and MAP values that were at or above target goals. 

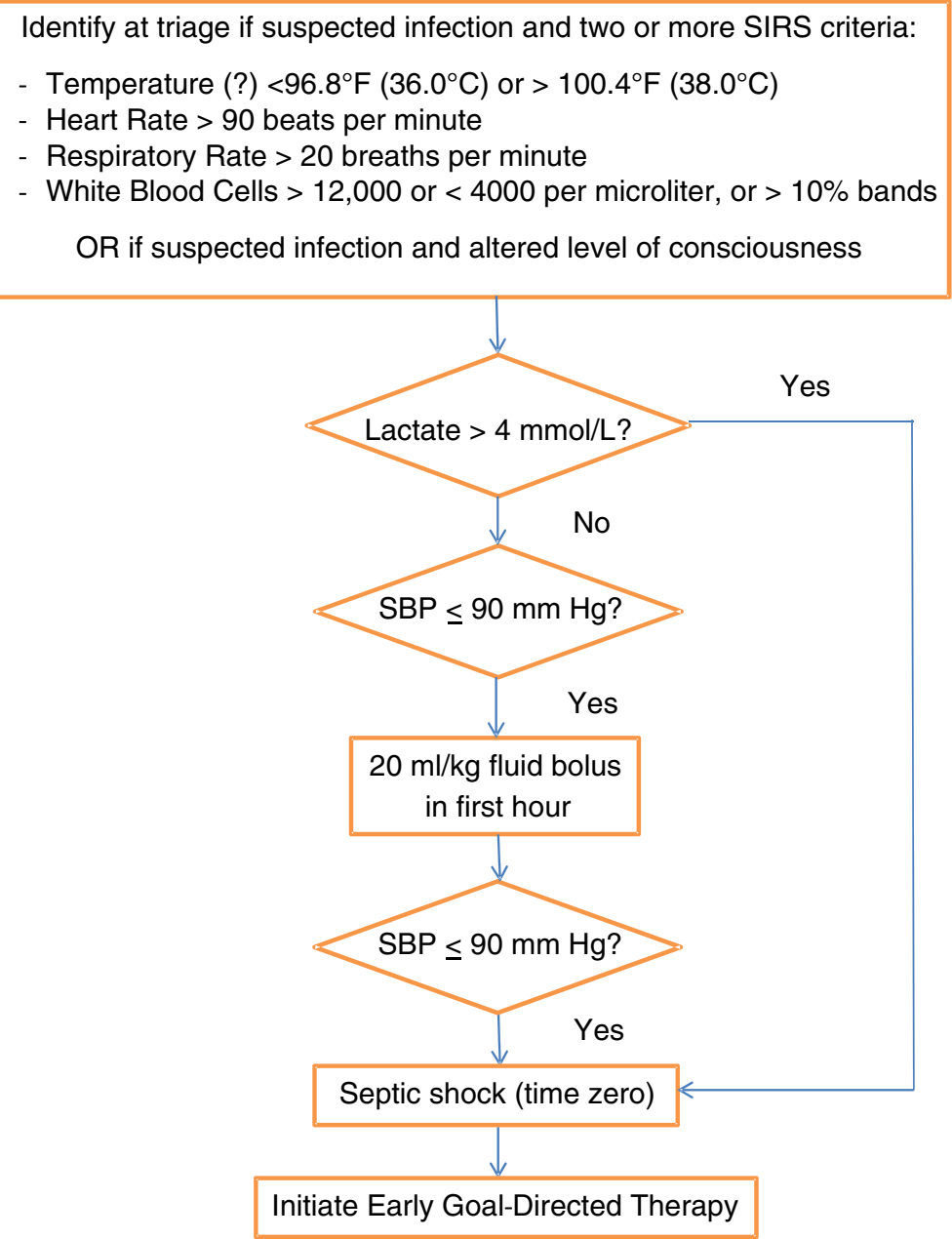

Figure 1 Early goal-directed therapy eligibility flowchart, Kaiser Permanente Northern California. Patients presenting with suspected infection and either two or more indicators of a systemic inflammatory response syndrome (SIRS) OR altered mental status are assessed for early goal-directed therapy eligibility based on both (1) initial venous lactate and (2) systolic blood pressure (SBP) as above. WBC, white blood cells.

Accepted $\mathrm{ScvO}_{2}$ measurements included those obtained by blood drawn from a central venous catheter and PreSep oximetry catheter values that were electronically documented in the patient's chart. This inclusion strategy was designed to identify patients with relatively clear indications for $\mathrm{ScvO}_{2}$-guided therapies as opposed to patients with a single low $\mathrm{ScvO}_{2}$ measurement, which may be spurious. Investigators blinded to the study hypothesis manually confirmed inclusion criteria and verified that inotropes (dobutamine, epinephrine or milrinone) and/or RBC transfusions were administered in response to $\mathrm{ScvO}_{2}$ measurements below $70 \%$ by examining patient-level collated electronic laboratory and hemodynamic data, including medication administration records, supplemented by clinical notes within the electronic health record. Patients under the age of 18 or with pregnancy were excluded from the prospective EGDT database.

\section{Methods and measurements}

Electronically abstracted variables from the first six hours of EGDT eligibility included age, initial heart rate, initial systolic and diastolic blood pressure, initial shock index, total intravenous fluids administered, time to initial antibiotic administration, initial measured $\mathrm{ScrO}_{2}$, final measured $\mathrm{ScvO}_{2}$ (minimum one-hour interval from the initial $\mathrm{ScvO}_{2}$ ), initial peripheral blood lactate, final peripheral blood lactate (minimum one-hour interval from the initial lactate), percent lactate clearance per hour (the average hourly change between the initial and final lactate measurements, where positive measurements reflect decreasing lactate values), initiation of mechanical ventilation, vasopressor administration (dopamine, norepinephrine, epinephrine, phenylephrine or vasopressin) and the lowest hemoglobin value (nadir). We categorized several continuous variables based on clinically relevant or previously published significant thresholds: final 


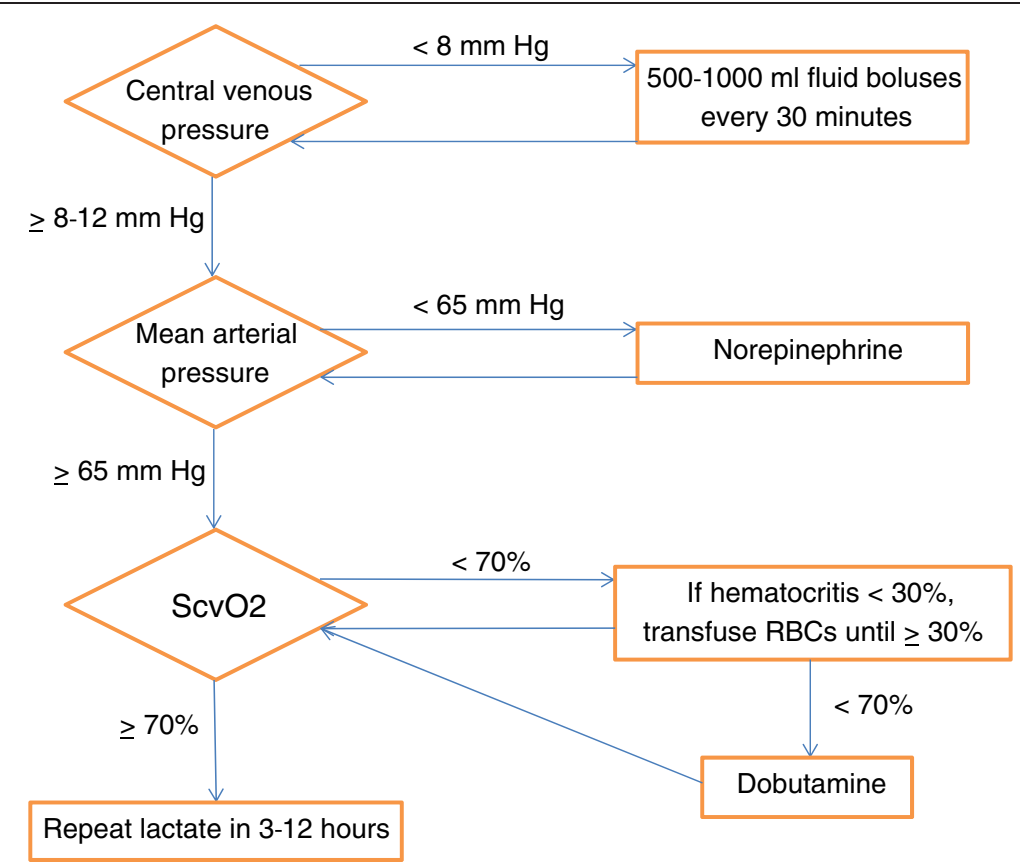

Figure 2 Early goal-directed therapy treatment flowchart, Kaiser Permanente Northern California. Patients are considered for central venous oxygenation saturation $\left(\mathrm{S}_{\mathrm{CVO}}\right)_{2}$-guided therapies once their central venous and mean arterial pressures have been optimized with fluid and vasopressor therapies as indicated. Patients with $\mathrm{ScvO}_{2}$ values less than $70 \%$ are transfused with red blood cells (RBCs) until the hematocrit is $30 \%$ or greater, followed by dobutamine administration if $\mathrm{ScvO}_{2}$ remains $<70 \%$.

$\mathrm{ScvO}_{2}<70 \%$ [1], shock index $\geq 1.0$ [37], lactate $\geq 4.0 \mathrm{mmol} / \mathrm{L}$ $[1,38]$, average hourly lactate clearance $\geq 5 \%[31]$ and antibiotic administration within one hour of shock recognition [39]. Hemoglobin values were abstracted as opposed to hematocrit values since clinicians often used a point-of-care test during resuscitation (iStat, Abbott Laboratories, Abbott Park, IL, USA) that reports hemoglobin but not hematocrit values. For the purpose of this study a hemoglobin value of $10 \mathrm{~g} / \mathrm{dL}$ was considered equivalent to a hematocrit of $30 \%$.

To enable case-mix adjustment we calculated the following morbidity and mortality prediction scores using previously validated approaches: (1) a modified Sequential Organ Failure Assessment (mSOFA) score [40], (2) a summary modified Elixhauser score [41] and (3) a KPNC-specific electronic Simplified Acute Physiology Score III (eSAPS 3) [42]. The mSOFA score was calculated using data obtained during the first six hours of EGDT eligibility (six-hour mSOFA score) and during the first 24 hours of hospitalization (24-hour mSOFA score). SOFA scores obtained during initial ED evaluations have previously been validated among patients with sepsis [43]. The summary modified Elixhauser score is a composite score based on International Classification of Diseases, Ninth Revision (ICD-9) codes for 30 categories of comorbidities. The eSAPS 3 score is a validated adaptation of the original SAPS 3 score using electronic data from KPNC patients collected one hour before and one hour following ICU admission. Together and individually the prediction scores demonstrated moderate discrimination when tested against in-hospital mortality rates in this dataset (combined model $\mathrm{c}$ statistic $=0.75$, individual model c statistics ranged from 0.65 to 0.71 ).

\section{Outcomes and analysis}

The primary outcome was in-hospital mortality. We examined the degree of association between the dependent variable (in-hospital mortality) and treatments of interest (inotrope administration and RBC transfusion), adjusting for covariates, using multivariate logistic regression [44]. After exclusion of collinearity based on an $r^{2}>0.7$, covariates with strong biologic plausibility or a potentially significant association with the dependent variable on bivariate analysis (defined as a $P$ value $\leq 0.1$ ) were included in the initial model, which was then modified in a stepwise fashion. Age and mortality prediction scores were further refined using cubic splines. The final regression model included age, sex, initial lactate, RBC transfusion, inotropic therapy, total intravenous fluids, hemoglobin nadir, vasopressor administration, initiation of mechanical ventilation, percent lactate clearance per hour, shock index, six-hour mSOFA score, Elixhauser score, and final $\mathrm{ScvO}_{2} \geq 70 \%$.

In order to further refine this analysis according to a primary indication for RBC transfusion (hemoglobin nadir less than 10 grams per deciliter $(\mathrm{g} / \mathrm{dL})$ ) or inotrope administration (hemoglobin nadir of $10 \mathrm{~g} / \mathrm{dL}$ or greater) 
for treatment of $\mathrm{ScvO}_{2}$ values $<70 \%$ (Figure 2), we divided the cohort based on hemoglobin nadir values during the first six hours of EGDT eligibility. We then determined separate propensity scores for $\mathrm{RBC}$ transfusion and inotrope administration using demographic, comorbidity, initial presenting and EGDT treatment period variables in a multivariable logistic regression model. Adjusted $P$ values were calculated by regressing the respective propensity score and treatment against each covariate (as the dependent variable). We did not pursue propensity matching given the limited numbers of study subjects. Rather, we performed logistic regression to assess for an association between treatment (with RBC transfusion or inotropic therapy) and the primary outcome within the respective subgroups, adjusting for the propensity score as both a continuous value and as an ordinal variable stratified into propensity quintiles [45]. Baseline or treatment variables with an adjusted $P$ value of $\leq 0.1$ were also included in the regression model as potential residual confounders. All final regression models have a minimum of five event outcomes per independent variable with at least thirty total events [46].

Continuous variables are reported as median values with interquartile ranges. Dichotomous variables are reported as percentages. All analyses were performed using Stata v13.1 (StataCorp LP, College Station, TX, USA). Categorical variables were analyzed using chi-square or Fisher's exact tests. Continuous variables were analyzed using the Student's $t$ test or Wilcoxon rank-sum test. Potential interactions between RBC transfusion, inotropic therapy, lactate clearance per hour (\%) and final $\mathrm{ScvO}_{2}$ values were analyzed as part of the modeling process.
Regression model results are reported as adjusted odds ratios (aOR) with robust 95\% confidence intervals (CIs) adjusted for clustering by hospital. Missing variables were imputed by multiple imputation $(n=5)$ using the mi command in Stata. Multicollinearity was assessed using the variance inflation factor (VIF). An alpha level of 0.05 was used as the threshold for statistical significance.

\section{Results}

There were 2,595 patient encounters in which EGDT was initiated in the ED during the 31-month study period. Of these, 579 met study inclusion criteria, of which seven were repeat encounters (that is readmissions), leaving a total of $572(22.0 \%)$ unique patient encounters eligible for study. A total of 312 ED physicians managed these 572 patients collectively, with mechanical ventilation initiated in $186(32.5 \%)$ patients and vasopressors administered during the treatment period in 335 (58.6\%) patients. A median of $4.0 \mathrm{~L}$ (interquartile range 3.0 to $5.0 \mathrm{~L}$ ) of intravenous fluids were given during the six-hour EGDT treatment period, with equal amounts administered to patients with a nadir hemoglobin $<10 \mathrm{~g} / \mathrm{dL}$ versus those with nadirs $\geq 10 \mathrm{~g} / \mathrm{dL}$ (4.0 $\mathrm{L}$ versus $4.0 \mathrm{~L}, P=0.09$ ). Ninety-three percent of patients $(534 / 572)$ had antibiotic therapy initiated within one hour of the septic shock recognition (time zero).

Either inotropic therapy or RBC transfusions were administered to 297 (51.9\%) study patients during the six-hour EGDT treatment period. Inotropic therapy was the sole treatment in 226 patients (39.5\%), RBC transfusion in 59 patients $(10.3 \%)$ and both interventions in 12 patients (2.1\%) (Figure 3). Among patients receiving

Encounters with EGDT initiated in the Emergency Department between March 2010 and September $2012(n=2595)$
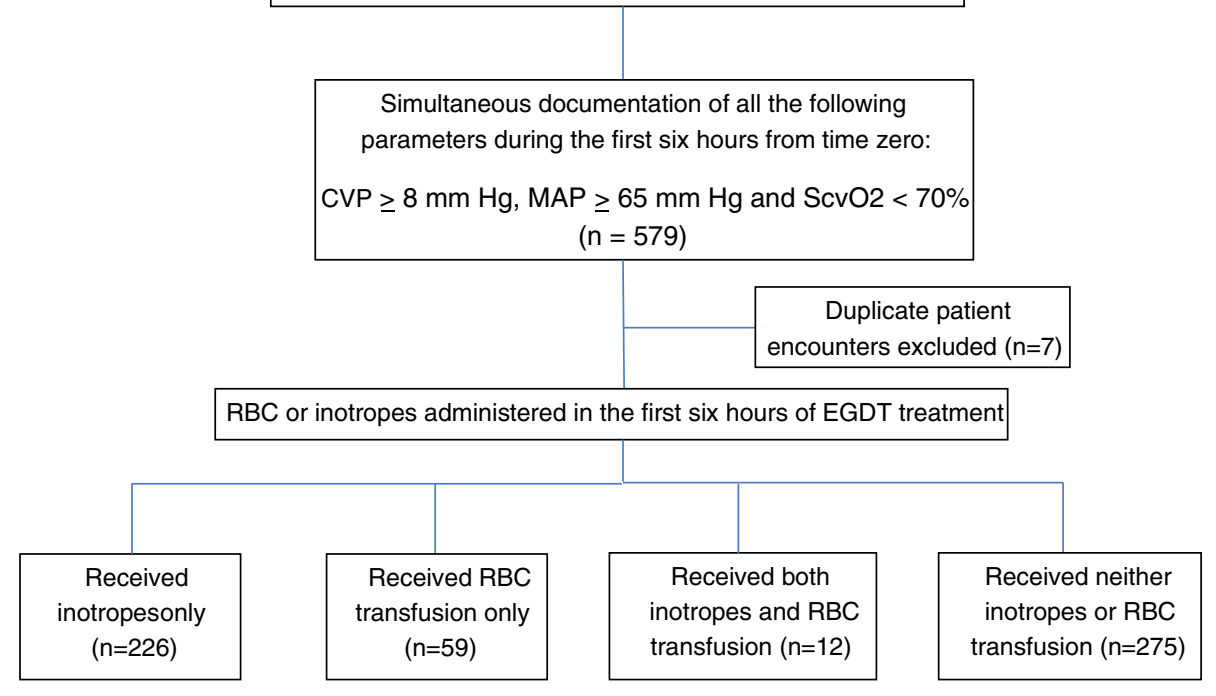

Figure 3 Study subject selection flowchart. CVP, central venous pressure; $\mathrm{DO}_{2}$, oxygen delivery; EGDT, early goal-directed therapy; MAP, mean arterial pressure; $\mathrm{RBC}$, red blood cell; $\mathrm{S}_{\mathrm{CVO}}$, central venous oxygen saturation. 
Table 1 Patient characteristics and variables according to $\mathrm{ScvO}_{2}$-guided therapies

\begin{tabular}{|c|c|c|c|c|}
\hline & $\begin{array}{l}\text { Entire cohort } \\
(n=572)\end{array}$ & $\begin{array}{l}\text { Inotrope administered } \\
(\mathrm{n}=238)\end{array}$ & $\begin{array}{l}\text { RBCs transfused } \\
(n=71)\end{array}$ & $\begin{array}{l}\text { No intervention } \\
(\mathrm{n}=275)\end{array}$ \\
\hline \multicolumn{5}{|l|}{ Demographics and comorbidities } \\
\hline Age, years & $73(62-82)$ & $76(65-83)$ & $70(61-81)$ & $71(60-81)$ \\
\hline Female (\%) & 46.7 & 47.1 & 46.5 & 46.2 \\
\hline \multicolumn{5}{|l|}{ Race } \\
\hline White (\%) & 57.9 & 57.1 & 49.3 & 59.6 \\
\hline Asian (\%) & 13.5 & 15.5 & 14.1 & 12.0 \\
\hline Hispanic (\%) & 12.9 & 11.8 & 14.1 & 13.5 \\
\hline African-American (\%) & 8.2 & 7.6 & 14.1 & 7.6 \\
\hline Other (\%) & 7.5 & 8.0 & 8.4 & 7.3 \\
\hline Ischemic heart disease (\%) & 31.3 & 30.7 & 38.0 & 30.2 \\
\hline Congestive heart failure (\%) & 36.4 & 39.5 & 32.4 & 34.2 \\
\hline Chronic lung disease (\%) & 34.1 & 30.7 & 26.7 & 38.9 \\
\hline Diabetes (\%) & 34.8 & 34.4 & 26.7 & 37.1 \\
\hline HIV/AIDS (\%) & 0.9 & 0.4 & 1.4 & 1.1 \\
\hline Malignancy (\%) & 14.9 & 12.2 & 32.4 & 13.1 \\
\hline Rheumatologic disease (\%) & 6.3 & 5.0 & 9.9 & 6.5 \\
\hline Elixhauser score & $16(8-23)$ & $16(10-22)$ & $19(11-28)$ & $15(7-23)$ \\
\hline \multicolumn{5}{|l|}{ Initial presenting variables } \\
\hline Systolic blood pressure, $\mathrm{mm} \mathrm{Hg}^{a}$ & $109(92-129)$ & $109(92-129)$ & $102(86-113)$ & $112(93-132)$ \\
\hline Diastolic blood pressure, $\mathrm{mm} \mathrm{Hg}^{a}$ & $63(52-76)$ & $63(50-76)$ & $56(44-68)$ & $64(53-77)$ \\
\hline Heart rate, $\mathrm{bpm}^{a}$ & $109(92-125)$ & $108(91-124)$ & $118(99-130)$ & $108(90-124)$ \\
\hline Shock index ${ }^{a}$ & $1.0(0.8-1.2)$ & $1.0(0.8-1.2)$ & $1.1(0.9-1.4)$ & $1.0(0.8-1.2)$ \\
\hline Initial serum lactate, $\mathrm{mmol} / \mathrm{L}^{b}$ & $4.4(3.0-6.2)$ & $4.5(3.2-6.3)$ & $4.4(3.6-7.7)$ & $4.3(2.8-6.0)$ \\
\hline Positive blood cultures (\%) & 35.3 & 35.7 & 49.3 & 32.4 \\
\hline \multicolumn{5}{|l|}{ EGDT treatment period variables } \\
\hline Fluid administered by 6 hours, liters & $4.0(3.0-5.0)$ & $4.0(3.0-5.1)$ & $4.1(3.0-5.0)$ & $4.0(2.6-5.0)$ \\
\hline Peak CVP, mm Hg & $16(13-21)$ & $16(13-21)$ & $15(12-19)$ & $16(13-22)$ \\
\hline Hemoglobin nadir, $\mathrm{g} / \mathrm{dL}^{b}$ & $11.4(9.5-13.2)$ & $12.1(10.3-13.6)$ & $7.9(7.1-8.8)$ & $11.3(10.0-13.1)$ \\
\hline Antibiotic therapy within one hour (\%) & 93.4 & 94.5 & 95.8 & 91.6 \\
\hline Mechanical ventilation $(\%)^{b}$ & 32.5 & 32.4 & 31.0 & 32.7 \\
\hline Vasopressor administration (\%) ${ }^{b}$ & 58.6 & 72.7 & 62.0 & 46.2 \\
\hline Initial $\mathrm{ScvO}_{2}$, percent ${ }^{b}$ & $63(55-69)$ & $62(55-69)$ & $61(55-68)$ & $64(57-69)$ \\
\hline Final $\mathrm{ScvO}_{2}$, percent ${ }^{b}$ & $68(61-73)$ & $68(62-73)$ & $70(60-75)$ & $67(61-72)$ \\
\hline Final $\mathrm{ScvO}_{2}>70 \%(\%)$ & 43.5 & 46.6 & 51.3 & 39.4 \\
\hline Lactate clearance, percent per hour ${ }^{b}$ & $8.4(4.5-12.9)$ & $8.3(4.2-12.6)$ & $9.5(3.6-13.0)$ & $8.3(4.5-13.0)$ \\
\hline Lactate clearance $>5 \%$ per hour (\%) & 72.5 & 72.5 & 72.4 & 71.8 \\
\hline mSOFA score at 6 hours & $4(3-6)$ & $5(4-7)$ & $5(3-6)$ & $4(2-6)$ \\
\hline ESAPS 3 score & $50(44-57)$ & $52(46-58)$ & $52(45-59)$ & $48(43-55)$ \\
\hline \multicolumn{5}{|l|}{ Post-treatment variables } \\
\hline mSOFA score at 24 hours & $5(3-7)$ & $5(4-7)$ & $5(3-7)$ & $4(3-6)$ \\
\hline In-hospital mortality (\%) & 20.5 & 22.7 & 18.3 & 19.3 \\
\hline
\end{tabular}

Continuous values are presented as median values with interquartile ranges in parentheses. Dichotomous variables are presented as percentages. ${ }^{a} \mathrm{At}$ initial emergency department presentation; ${ }^{b}$ within the first six hours from shock recognition (time zero). $\mathrm{ScvO}_{2}$, central venous oxygen saturation; RBC, red blood cells; $\mathrm{HIV/AIDS}$, human immunodeficiency virus/acquired immunodeficiency syndrome; $\mathrm{mm} \mathrm{Hg}$, millimeters mercury; bpm, beats per minute; EGDT, early goal-directed therapy; CVP, central venous pressure; $\mathrm{g} / \mathrm{dL}$, grams per deciliter; mSOFA, modified sequential organ failure assessment score; eSAPS, electronic simplified acute physiology score. 
Table 2 Propensity score adjustment among patients eligible for RBC transfusion during EGDT

\begin{tabular}{|c|c|c|c|c|}
\hline & RBCs transfused $(n=71)$ & No RBCs transfused $(n=108)$ & $P$ value (unadjusted) & $P$ value (adjusted) \\
\hline \multicolumn{5}{|l|}{ Demographics and comorbidities } \\
\hline Age, years & $70(61-81)$ & $72(60-84)$ & 0.78 & 0.84 \\
\hline Female (\%) & 46.5 & 50.0 & 0.65 & 0.83 \\
\hline Race & & & 0.56 & 0.45 \\
\hline White (\%) & 49.3 & 53.7 & & \\
\hline Asian (\%) & 14.1 & 13.9 & & \\
\hline Hispanic (\%) & 14.1 & 14.8 & & \\
\hline African-American (\%) & 14.1 & 7.4 & & \\
\hline Other (\%) & 8.4 & 10.2 & & \\
\hline Ischemic heart disease (\%) & 38.0 & 28.7 & 0.19 & 0.38 \\
\hline Congestive heart failure (\%) & 32.4 & 31.4 & 0.90 & 0.67 \\
\hline Chronic lung disease (\%) & 26.7 & 25.9 & 0.90 & 0.87 \\
\hline Diabetes (\%) & 26.7 & 40.7 & 0.06 & 0.90 \\
\hline HIV/AIDS (\%) & 1.4 & 0.9 & 0.77 & 0.71 \\
\hline Malignancy (\%) & 32.4 & 15.7 & 0.01 & 0.93 \\
\hline Rheumatologic disease (\%) & 9.9 & 6.5 & 0.41 & 0.51 \\
\hline Elixhauser score & $19(11-28)$ & $16(9-24)$ & 0.05 & 0.95 \\
\hline \multicolumn{5}{|l|}{ Initial presenting variables } \\
\hline Systolic blood pressure, $\mathrm{mm} \mathrm{Hg}^{a}$ & $102(86-113)$ & $107(92-127)$ & 0.05 & 0.90 \\
\hline Diastolic blood pressure, $\mathrm{mm} \mathrm{Hg}^{a}$ & $56(44-68)$ & $59(50-70)$ & 0.51 & 0.54 \\
\hline Heart rate, bpm ${ }^{a}$ & $118(99-130)$ & $109(90-123)$ & 0.02 & 0.21 \\
\hline Shock index ${ }^{a}$ & $1.1(0.9-1.4)$ & $1.0(0.8-1.2)$ & 0.001 & 0.27 \\
\hline Shock index $\geq 1.0$ & 74.6 & 51.9 & 0.003 & 0.11 \\
\hline Serum lactate, $\mathrm{mmol} / \mathrm{L}^{b}$ & $4.4(3.6-7.7)$ & $4.3(2.5-5.7)$ & 0.10 & 0.74 \\
\hline Positive blood cultures (\%) & 49.3 & 34.2 & 0.05 & 0.73 \\
\hline \multicolumn{5}{|l|}{ EGDT treatment period variables } \\
\hline Fluid administered by 6 hours, liters & $4.1(3.0-5.0)$ & $4.0(3.0-5.7)$ & 0.37 & 0.48 \\
\hline Peak CVP, mm Hg & $15(12-19)$ & $16(13-22)$ & 0.02 & 0.57 \\
\hline Hemoglobin nadir, $\mathrm{g} / \mathrm{dL}^{b}$ & $7.9(7.1-8.8)$ & $9.2(8.5-9.5)$ & $<0.001$ & 0.47 \\
\hline Inotropes (\%) & 16.9 & 37.0 & 0.005 & 0.36 \\
\hline Antibiotic therapy within one hour (\%) & 95.8 & 93.5 & 0.52 & 0.40 \\
\hline Mechanical ventilation $(\%)^{b}$ & 31.0 & 33.3 & 0.74 & 0.74 \\
\hline Vasopressor administration (\%) & 62.0 & 64.8 & 0.70 & 0.60 \\
\hline Initial $\mathrm{ScvO}_{2}$, percent ${ }^{b}$ & $61(55-68)$ & $63(55-69)$ & 0.97 & 0.87 \\
\hline Final $\mathrm{ScvO}_{2}$, percent ${ }^{b}$ & $70(60-75)$ & $67(61-71)$ & 0.51 & 0.36 \\
\hline Final $\mathrm{ScvO}_{2}>70 \%(\%)$ & 51.3 & 39.1 & 0.12 & 0.16 \\
\hline Change in $\mathrm{ScvO}_{2}$, percent & $7(-2-12)$ & $5(-4-12)$ & 0.54 & 0.34 \\
\hline Lactate clearance, percent per hour ${ }^{b}$ & $9.5(3.6-13.0)$ & $8.5(3.9-12.0)$ & 0.13 & 0.09 \\
\hline Lactate clearance $\geq 5 \%$ per hour (\%) & 72.4 & 66.5 & 0.43 & 0.28 \\
\hline mSOFA score at 6 hours & $5(3-6)$ & $5(3-7)$ & 0.41 & 0.94 \\
\hline eSAPS 3 score & $52(45-59)$ & $50(44-57)$ & 0.35 & 0.89 \\
\hline
\end{tabular}


Table 2 Propensity score adjustment among patients eligible for RBC transfusion during EGDT (Continued)

\begin{tabular}{|c|c|c|c|c|}
\hline \multicolumn{5}{|l|}{ Post-treatment variables } \\
\hline mSOFA score at 24 hours & $5(3-7)$ & $5(4-7)$ & 0.38 & 0.85 \\
\hline In-hospital mortality (\%) & 18.3 & 19.4 & 0.85 & 0.12 \\
\hline \multicolumn{5}{|c|}{$\begin{array}{l}\text { A subgroup analysis was conducted among patients with a hemoglobin nadir less than } 10 \mathrm{~g} / \mathrm{dL} \text { during EGDT. Unadjusted } P \text { values are reported for the observed } \\
\text { variables based on whether RBC transfusion was administered for an } \mathrm{ScvO} 2 \text { less than } 70 \% \text {. Adjusted } P \text { values are based on bivariate regression analyses } \\
\text { comparing the propensity score (for RBC transfusion) and each variable. Continuous values are presented as median values with interquartile ranges in } \\
\text { parentheses. Dichotomous variables are presented as percentages. } \\
{ }^{a} \text { At initial emergency department presentation; }{ }^{b} \text { within the first six hours from shock recognition (time zero). RBC, red blood cells; EGDT, early goal-directed } \\
\text { therapy; HIV/AIDS, human immunodeficiency virus/acquired immunodeficiency syndrome; mm Hg, millimeters mercury; bpm, beats per minute; CVP, central } \\
\text { venous pressure; g/dL, grams per deciliter; } \mathrm{ScvO}_{2} \text {, central venous oxygen saturation; mSOFA, modified sequential organ failure assessment score; eSAPS, electronic } \\
\text { simplified acute physiology score. }\end{array}$} \\
\hline
\end{tabular}

inotropic therapy, dobutamine was the sole inotrope in 237 (99.6\%) patients (consistent with the KPNC EGDT treatment protocol, Figure 2), with a single additional patient receiving an epinephrine infusion. Median values for age, initial lactate and initial $\mathrm{ScvO}_{2}$ were 73 years, $4.4 \mathrm{mmol} / \mathrm{L}$ and $63 \%$, respectively. A total of $249 / 572$ (43.5\%) patients reached a final ScvO2 of $\geq 70 \%$ by the end of the six-hour EGDT treatment period. Patients were not statistically more likely to achieve an $\mathrm{ScvO}_{2}$ of $\geq 70 \%$ if they were treated with RBC transfusion alone (29/59, $49.2 \%, P=0.19)$, inotropic therapy alone $(104 / 226,46.0 \%$, $P=0.15)$ or both RBC and inotropic therapy $(7 / 12,58.3 \%$, $P=0.23)$ as compared to no therapy $(108 / 275,39.3 \%)$. There was no clinically significant unadjusted difference in the rate of lactate clearance according to treatment provided. It should be noted that the small number of patients receiving both $\mathrm{RBC}$ and inotropic therapy limits meaningful analysis of this subgroup. A complete list of abstracted variables grouped by treatment with RBC transfusion or inotropic therapy is presented in Table 1.

There were no significant unadjusted associations between in-hospital mortality and either inotropic therapy (OR 1.33, 95\% CI 0.92 to $1.92, P=0.12$ ) or RBC transfusion (OR $0.93,95 \%$ CI 0.52 to $1.66, P=0.80$ ). Multiple logistic regression among the entire cohort adjusted for age, sex, initial lactate, RBC transfusion, inotropic therapy, total intravenous fluids, hemoglobin nadir, vasopressor administration, initiation of mechanical ventilation, percent lactate clearance per hour, shock index, six-hour mSOFA score, Elixhauser score and final $\mathrm{ScvO}_{2} \geq 70 \%$ likewise did not reveal a statistically significant association between in-hospital mortality and inotropic therapy (aOR 1.10, 95\% CI 0.70 to $1.73, P=0.68$ ), but did suggest a trend toward lower mortality among patients transfused with RBCs (aOR 0.58, 95\% CI 0.29 to 1.18 , $P=0.14)$. Pairwise factorial interactions between $\mathrm{RBC}$ transfusion, inotropic therapy, final $\mathrm{ScvO}_{2} \geq 70 \%$ or lactate clearance $\geq 5 \%$ per hour were not significantly associated with in-hospital mortality when introduced into the regression model, nor did they significantly alter the odds ratio estimates for $\mathrm{RBC}$ transfusion or inotropic therapy, suggesting a treatment effect that was independent of reaching these resuscitation endpoints. The VIF was less than four for all independent variables, indicating the absence of multicollinearity,

We then conducted a propensity-adjusted analysis for treatment with RBC transfusion or inotropic therapy among subgroups of patients with a hemoglobin nadir of either less than $10 \mathrm{~g} / \mathrm{dL}$ or equal or greater than $10 \mathrm{~g} / \mathrm{dL}$, respectively. The subgroup characteristics are presented in Tables 2 and 3, stratified by the treatments of interest, along with the covariate $P$ values following adjustment for treatment propensity score. The propensity-adjusted regression model estimated that $\mathrm{RBC}$ transfusion was significantly associated with decreased in-hospital mortality (aOR 0.42 , 95\% CI 0.18 to $0.97, P=0.04$ ) among patients with a hemoglobin nadir less than $10 \mathrm{~g} / \mathrm{dL}$. However, inotropic therapy was not associated with in-hospital mortality (aOR $1.16,95 \%$ CI 0.69 to $1.96, P=0.57$ ). These results were largely consistent when the model was adjusted using quintiles of propensity score, though the point estimate and CIs were slightly higher resulting in a marginally non-significant result for $\mathrm{RBC}$ transfusion (aOR 0.46, 95\% CI 0.21 to $1.04, P=0.06$, Table 4 ).

\section{Discussion}

In this retrospective analysis of a prospectively identified patient cohort with septic shock undergoing EGDT in the emergency department, $\mathrm{DO}_{2}$ augmentation therapies (inotropes and/or RBC transfusion) were administered to just over half the patients with EGDT protocol-driven indications for these treatments $\left(\mathrm{ScvO}_{2}<70 \%\right.$ after meeting both CVP and MAP target goals). We observed a propensity-adjusted association between $\mathrm{RBC}$ transfusion and decreased in-hospital mortality in patients with hemoglobin values less than $10 \mathrm{~g} / \mathrm{dL}$, raising the possibility of a beneficial effect of RBC transfusion as recommended by the EGDT treatment protocol. This association was not significantly impacted by the success or failure of reaching a final $\mathrm{ScvO}_{2}$ of $\geq 70 \%$. In contrast, analysis of patients receiving inotropic therapy alone did not reveal a significant association with in-hospital mortality rates. These findings may be interpreted to suggest a mortality benefit from $\mathrm{RBC}$ transfusion among a specific subgroup of 
Table 3 Propensity score adjustment among patients eligible for inotropic therapy during EGDT

\begin{tabular}{|c|c|c|c|c|}
\hline & $\begin{array}{l}\text { Inotropes } \\
(n=186)\end{array}$ & $\begin{array}{l}\text { No inotropes } \\
(n=207)\end{array}$ & $P$ value (unadjusted) & $\begin{array}{l}P \text { value (adjusted } \\
\text { for propensity score) }\end{array}$ \\
\hline \multicolumn{5}{|l|}{ Demographics and comorbidities } \\
\hline Age, years & $76(67-84)$ & $72(61-81)$ & 0.01 & 0.85 \\
\hline Female (\%) & 46.8 & 44.9 & 0.74 & 0.58 \\
\hline Race & & & 0.45 & 0.47 \\
\hline White (\%) & 58.6 & 62.3 & & \\
\hline Asian (\%) & 15.1 & 11.6 & & \\
\hline Hispanic (\%) & 11.8 & 12.6 & & \\
\hline African-American (\%) & 6.4 & 8.2 & & \\
\hline Other (\%) & 8.0 & 5.3 & & \\
\hline Ischemic heart disease (\%) & 31.7 & 30.0 & 0.38 & 0.96 \\
\hline Congestive heart failure (\%) & 39.8 & 37.2 & 0.60 & 0.91 \\
\hline Chronic lung disease (\%) & 32.2 & 42.5 & 0.04 & 0.49 \\
\hline Diabetes (\%) & 34.4 & 34.8 & 0.94 & 0.29 \\
\hline HIV/AIDS (\%) & 0.5 & 1.0 & 0.63 & 0.66 \\
\hline Malignancy (\%) & 10.8 & 12.1 & 0.68 & 0.73 \\
\hline Rheumatologic disease (\%) & 4.8 & 6.3 & 0.54 & 0.57 \\
\hline Elixhauser score & $16(8-21)$ & $15(7-23)$ & 0.64 & 0.38 \\
\hline \multicolumn{5}{|l|}{ Initial presenting variables } \\
\hline Systolic blood pressure, $\mathrm{mm} \mathrm{Hg}^{a}$ & $111(94-130)$ & $113(95-132)$ & 0.57 & 0.23 \\
\hline Diastolic blood pressure, $\mathrm{mm} \mathrm{Hg}^{a}$ & $65(51-80)$ & $66(54-78)$ & 0.51 & 0.31 \\
\hline Heart rate, bpm ${ }^{a}$ & $108(92-124)$ & $108(90-125)$ & 0.80 & 0.50 \\
\hline Shock index ${ }^{a}$ & $1.0(0.8-1.2)$ & $1.0(0.8-1.1)$ & 0.85 & 0.94 \\
\hline Shock index $\geq 1.0$ & 52.7 & 50.2 & 0.63 & 0.74 \\
\hline Serum lactate, $\mathrm{mmol} / \mathrm{L}^{b}$ & $4.6(3.4-6.2)$ & $4.4(3.0-6.2)$ & 0.22 & 0.47 \\
\hline Positive initial blood cultures (\%) & 34.4 & 31.9 & 0.60 & 0.40 \\
\hline \multicolumn{5}{|l|}{ EGDT treatment period variables } \\
\hline Fluid administered by 6 hours, liters & $4.0(3.0-5.0)$ & $3.5(2.5-5.0)$ & 0.03 & 0.34 \\
\hline Peak CVP, $\mathrm{mm} \mathrm{Hg}^{b}$ & $16(13-20)$ & $17(13-22)$ & 0.27 & 0.45 \\
\hline Hemoglobin nadir, $\mathrm{g} / \mathrm{dL}^{b}$ & $12.7(11.6-14.0)$ & $12.2(10.9-13.7)$ & 0.04 & 0.11 \\
\hline RBC transfusion (\%) & 0 & 0 & $\mathrm{n} / \mathrm{a}$ & $\mathrm{n} / \mathrm{a}$ \\
\hline Antibiotic therapy within one hour (\%) & 95.2 & 90.8 & 0.10 & 0.11 \\
\hline Mechanical ventilation $(\%)^{b}$ & 30.6 & 34.3 & 0.44 & 0.58 \\
\hline Vasopressor administration (\%) ${ }^{b}$ & 71.5 & 42.5 & $<0.001$ & 0.31 \\
\hline Initial $\mathrm{ScvO}_{2}$, percent ${ }^{b}$ & $62(55-70)$ & $64(57-69)$ & 0.44 & 0.07 \\
\hline Final $\mathrm{ScvO}_{2}$, percent ${ }^{b}$ & $68(63-72)$ & $68(61-73)$ & 0.29 & 0.62 \\
\hline Final $\mathrm{ScvO}_{2}>70 \%(\%)$ & 44.4 & 42.3 & 0.68 & 0.80 \\
\hline Change in $\mathrm{ScvO}_{2}$, percent & $5(-2-13)$ & $3(-4-10)$ & 0.16 & 0.06 \\
\hline Lactate clearance, percent per hour ${ }^{b}$ & $8.2(4.8-13.0)$ & $8.5(4.8-13.6)$ & 0.40 & 0.38 \\
\hline Lactate clearance $\geq 5 \%$ per hour (\%) & 74.1 & 74.2 & 0.98 & 0.89 \\
\hline mSOFA score at 6 hours & $5(4-6)$ & $4(2-6)$ & $<0.001$ & 0.51 \\
\hline eSAPS 3 score & $52(46-58)$ & $48(43-55)$ & 0.02 & 0.71 \\
\hline
\end{tabular}


Table 3 Propensity score adjustment among patients eligible for inotropic therapy during EGDT (Continued)

\begin{tabular}{|c|c|c|c|c|}
\hline \multicolumn{5}{|l|}{ Post-treatment variables } \\
\hline mSOFA score at 24 hours & $5(4-7)$ & $4(3-6)$ & 0.001 & 0.21 \\
\hline In-hospital mortality (\%) & 23.7 & 18.8 & 0.24 & 0.41 \\
\hline \multicolumn{5}{|c|}{$\begin{array}{l}\text { A subgroup analysis was conducted among patients with a hemoglobin nadir of } 10 \mathrm{~g} / \mathrm{dL} \text { or greater during EGDT. Unadjusted } P \text { values are reported for the } \\
\text { observed variables based on whether inotropic therapy was administered for an } \mathrm{ScvO} 2 \text { less than } 70 \% \text {. Adjusted } P \text { values are based on bivariate regression } \\
\text { analyses comparing the propensity score (for inotropic therapy) and each variable. Continuous values are presented as median values with interquartile ranges in } \\
\text { parentheses. Dichotomous variables are presented as percentages. } \\
{ }^{a} \text { At initial emergency department presentation; }{ }^{b} \text { within the first six hours from shock recognition (time zero). ; EGDT, early goal-directed therapy; HIV/AIDS, human } \\
\text { immunodeficiency virus/acquired immunodeficiency syndrome; } \mathrm{mm} \mathrm{Hg} \text {, millimeters mercury; bpm, beats per minute; CVP, central venous pressure; g/dL, grams } \\
\text { per deciliter; RBC, red blood cells; } \mathrm{ScvO}_{2} \text {, central venous oxygen saturation; mSOFA, modified sequential organ failure assessment score; eSAPS, electronic } \\
\text { simplified acute physiology score. }\end{array}$} \\
\hline
\end{tabular}

patients (subnormal $\mathrm{ScvO}_{2}$ and hemoglobin less than $10 \mathrm{~g} / \mathrm{dL}$ ) treated early in the course of septic shock, independent of reaching a target $\mathrm{ScvO}_{2}$.

Our study is the first we are aware of to revisit the hypothetical independent benefit of RBC transfusion among patients with septic shock treated in the emergency department since the original EGDT trial [1]. RBC transfusion is arguably the most controversial aspect of the EGDT protocol for several reasons. Foremost, there is controversy over the use of RBC transfusions as a means of augmenting tissue oxygen delivery or microvascular perfusion in patients with sepsis $[16,18]$. Second, there is a fairly consistent and growing body of evidence demonstrating mortality benefits of restrictive transfusion practices in critical and acute illness, though not specifically among patients with septic shock [19-23]. Finally, the exceptionally high rate of transfusion (64\%) during the initial six hours of therapy in the original EGDT study [1] has failed to be approximated in any subsequent randomized or observational study of EGDT $[10,31-35,47]$.

However, several recent studies $[15,24,48]$ have challenged the notion that RBC transfusion is harmful in critically ill patients, and have even suggested a mortality benefit among septic patients using propensity-matched analyses, similar to our results $[15,24]$. Reasons for the observed decreases in mortality following RBC transfusion during septic shock are elusive at best, though may involve the aforementioned contested theory of augmented microvascular oxygen delivery and the interplay between the pro-inflammatory and immunosuppressive effects of blood transfusions [49]. Others have suggested the increasing use of leukoreduced products in the past decade as a possible explanation [48]. Accordingly, physiologic studies of leukoreduced RBC transfusions in patients with sepsis have revealed improvements in microcirculatory flow and oxygen consumption, chiefly among those with subnormal parameters at baseline $[18,50]$. In addition, the pro-inflammatory effects of leukoreduced blood, as compared to non-leukoreduced blood, appear to be minimal given near undetectable levels of cytokines, suggesting a possible mechanism by which non-leukoreduced products produced harmful effects in older studies [51].

The lack of an observed association between inotropic therapy and in-hospital mortality is consistent with previously published findings from a similar multicenter sepsis patient database analysis [9]. This is despite the fact that the patients treated with inotropic therapy in this cohort received a larger volume of intravenous fluid than those who were not treated with inotropes (median 4.0 versus 3.5 liters, $P=0.03$ ). Some studies have even hinted at potential harms resulting from the use of dobutamine in patients with septic shock $[13,29]$. In part this may be attributable to the highly variable physiologic effects of dobutamine among patients with severe sepsis or septic shock, specifically with regard to indices of microvascular perfusion, even in the presence of hypoperfusion [26-28]. Biochemical studies in septic shock patients have demonstrated positive relationships between dobutamine administration and levels of the pro-inflammatory cytokine tumor

Table 4 Multiple regression and propensity score adjusted odds ratio of in-hospital mortality according to treatment with red blood cell (RBC) transfusion or inotropic therapy during early goal-directed therapy (EGDT)

\begin{tabular}{|c|c|c|c|c|}
\hline Odds ratio of in-patient mortality & RBC transfusion & $P$ value & Inotrope therapy & $P$ value \\
\hline Unadjusted (95\% Cl) & $0.93(0.52-1.66)$ & 0.80 & $1.33(0.92-1.92)$ & 0.12 \\
\hline Multivariate regression adjusted (95\% Cl) & $0.58(0.29-1.18)$ & 0.14 & $1.10(0.70-1.73)$ & 0.68 \\
\hline Propensity score (continuous) adjusted (95\% Cl) & $0.42(0.18-0.97)$ & 0.04 & $1.16(0.69-1.96)$ & 0.57 \\
\hline Propensity score (quintile) adjusted (95\% Cl) & $0.46(0.21-1.04)$ & 0.06 & $1.21(0.77-1.91)$ & 0.41 \\
\hline
\end{tabular}

Multivariate logistic regression analysis of the entire cohort was conducted, followed by propensity score adjusted analyses according to the subgroup of hemoglobin nadir during the first six hours of EGDT treatment; patients with a hemoglobin less than $10 \mathrm{~g} / \mathrm{dL}$ were included in the analysis of RBC transfusion and patients with a hemoglobin of $10 \mathrm{~g} / \mathrm{dL}$ or greater were included in the inotrope therapy analysis. Standard error and $95 \%$ confidence interval (Cl) calculations in the regression analyses are adjusted for clustering by hospital. 
necrosis factor (TNF)-alpha that are not explained by illness severity, and which may predispose patients to higher degrees of organ failure [52,53]. Finally, a recent clinical trial of beta-adrenergic receptor blockade during septic shock demonstrated an unexpected large mortality benefit, potentially unmasking the harms of excessive beta-receptor stimulation with inotropic therapy during septic shock [54].

The fact that only $43.5 \%$ of the patients in the study cohort successfully reached a final $\mathrm{ScvO}_{2}$ of $\geq 70 \%$ highlights the difficulty of translating evidence from rigorous clinical trials into general clinical practice (given that $95 \%$ versus $60 \%$ of patients met this goal in the experimental and control arms of the original EGDT trial, respectively) [1,55]. Regardless, we did not observe any strong correlations between the specific use of inotropes or RBC transfusions and the likelihood of reaching the $\mathrm{ScvO}_{2}$ endpoint, though it is difficult to interpret this finding given the possibility of unmeasured co-interventions and the small number of patients receiving both interventions during the resuscitation period.

\section{Limitations}

As with all retrospective studies, several variables of interest suffer from a lack of standardized data collection. Accordingly, it should be noted that analysis of this data is limited by the possibility of residual confounding or indication bias not accounted for by propensity adjustment. In particular, the lack of consistent intervals for repeated measurements is a notable limitation, most pertinent for the two study inclusion $\mathrm{ScvO} 2$ values, the percent lactate clearance and the final $\mathrm{ScvO}_{2}$ at the end of the six-hour EGDT treatment period. Since the observed associations between treatment with RBC transfusion or inotropic therapy and in-hospital mortality appear to be largely independent of these measurements this is likely of minor consequence. However, it is possible that the observed associations between treatment and outcomes are more accurately represented by achievement of these resuscitation endpoints than our data suggests.

We also did not control for the use of downstream interventions when adjusting for predictors of in-patient mortality (that is, use of corticosteroids and protective lung ventilation). However, protective lung ventilation is standard practice throughout our medical system with default ventilator orders for pressure-limited, low tidal volume parameters, so significant non-compliance was unlikely. Given that the independent impact of corticosteroid administration on mortality in sepsis is inconclusive [56-59], the omission of this covariate is unlikely to have altered our results significantly.

Likewise we did not fully control for the intensity of treatment with vasopressors or inotropic therapy. The former was partially adjusted for through the use of the cardiovascular component of the mSOFA score. Regarding inotropic therapy, however, it is possible that the failure to observe an association with in-hospital mortality may simply reflect inadequate dosing or titration. This is suggested by the marginal improvement in $\mathrm{ScvO}_{2}$ values observed in the inotropic therapy treatment group. Accordingly, it should be noted that this study was not designed to validate or repudiate the physiologic concepts underlying EGDT.

\section{Conclusions}

Among patients with septic shock treated with EGDT in the setting of subnormal $\mathrm{ScvO}_{2}$ values despite meeting CVP and MAP target goals, treatment with RBC transfusion may be independently associated with decreased inhospital mortality. Treatment with inotropic therapy does not appear to be significantly associated with in-hospital mortality, though conclusions are limited by lack of adjustment for intensity of treatment. Randomized studies addressing the utility of specific $\mathrm{ScvO}_{2}$-guided therapies would be useful to both address controversial elements of EGDT and further enable septic shock treatment protocols to be refined for optimal efficacy and efficiency.

\section{Key messages}

- $\mathrm{DO}_{2}$ augmentation therapies (RBC transfusion or inotropic therapy) were not consistently given to patients with subnormal $\mathrm{ScrO}_{2}$ values in this EGDT patient cohort

- $\mathrm{RBC}$ transfusion for subnormal $\mathrm{ScvO}_{2}$ values $(<70 \%)$ during EGDT appears to be at least marginally associated with decreased in-hospital mortality, independent of meeting the $\mathrm{ScvO}_{2}$ target goal of 70\%

- The administration of inotropic therapy for subnormal $\mathrm{ScvO}_{2}$ values during EGDT does not appear to be associated with reduced in-hospital mortality

- Randomized studies of RBC transfusion in early septic shock are needed.

\section{Abbreviations}

aOR: adjusted odds ratio; bpm: beats per minute; Cl: confidence interval; CVP: central venous pressure; $\mathrm{DO}_{2}$ : oxygen delivery; $\mathrm{ED}$ : emergency department; EGDT: early goal-directed therapy; eSAPS 3: electronic simplified acute physiology score III; g/dL: grams per deciliter; HIV/AIDS: human immunodeficiency virus/acquired immunodeficiency syndrome;

IRB: institutional review board; KPNC: Kaiser Permanente Northern California; MAP: mean arterial pressure; mm Hg: millimeters mercury; mSOFA: modified sequential organ failure assessment score; $\mathrm{RBC}$ : red blood cell; $\mathrm{ScvO}_{2}$ : central venous oxygen saturation; $\mathrm{SpO}_{2}$ : peripheral blood oxygen saturation; TNF: tumor necrosis factor; VIF: variance inflation factor; $\mathrm{VO}_{2}$ : oxygen consumption.

\section{Competing interests}

The authors declare that they have no competing interests. 


\section{Authors' contributions}

DGM, JWM, ARE, MVK, VL, DWB, and DRV made substantial contributions to the conception and design of the study. DGM, ARE, MVK, and JWM refined participant and variable selection and undertook chart abstraction. YYH and DGM managed the data, including quality control, and performed data analysis. DGM drafted the manuscript, and all authors contributed substantially to its revision. All authors read and approved the final manuscript. DGM takes responsibility for the manuscript as a whole.

\section{Acknowledgements}

We would like to thank the Data Consulting Team of the Quality and AR\&L Division of Kaiser Foundation Hospital, Inc. for their partnership in data acquisition, Gabriel Escobar, MD for assistance with electronic simplified acute physiology score calculation, Adina S. Rauchwerger, MPH, Uli Chettipally, MD, MPH and Mary E. Reed, DrPH for their assistance with manuscript revision, Lorelle Poropat of the KPNC Quality and Regulatory Services Data Consulting Team and Jay Soule of the Quality and Operations Support division of The Permanente Medical Group for their help with case identification. The Gordon and Betty Moore Foundation provided the financial support for all authors involved in this project. The funding body had no role in the design, data collection, analysis or interpretation of the data, writing of the manuscript or the decision to submit the manuscript for publication.

\section{Author details}

'Department of Emergency Medicine, Kaiser Permanente, 275 West Macarthur Boulevard, Oakland, CA 94611, USA. ${ }^{2}$ Division of Research, Kaiser Permanente Northern California, 2000 Broadway, Oakland, CA 94612, USA. ${ }^{3}$ Department of Emergency Medicine, Kaiser Permanente, 27303 Sleepy Hollow Avenue, Hayward, CA 94545, USA. ${ }^{4}$ Department of Emergency Medicine, Kaiser Permanente, 6600 Bruceville Road, South Sacramento, CA 95823, USA. ${ }^{5}$ Department of Emergency Medicine, Kaiser Permanente, 99 Montecillo Road, San Rafael, CA 94903, USA. ${ }^{6}$ Department of Emergency Medicine, Kaiser Permanente, 1600 Eureka Road, Roseville, CA 95661, USA.

Received: 6 March 2014 Accepted: 13 August 2014

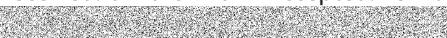

\section{References}

1. Rivers E, Nguyen B, Havstad S, Ressler J, Muzzin A, Knoblich B, Peterson E, Tomlanovich M: Early goal-directed therapy in the treatment of severe sepsis and septic shock. N Engl J Med 2001, 345:1368-1377.

2. Dellinger RP, Levy MM, Rhodes A, Annane D, Gerlach H, Opal SM, Sevransky JE, Sprung CL, Douglas IS, Jaeschke R, Osborn TM, Nunnally ME, Townsend SR, Reinhart K, Kleinpell RM, Angus DC, Deutschman CS, Machado FR, Rubenfeld GD, Webb SA, Beale RJ, Vincent JL, Moreno R: Surviving sepsis campaign: international guidelines for management of severe sepsis and septic shock: 2012. Crit Care Med 2013, 41:580-637.

3. Walley KR: Use of central venous oxygen saturation to guide therapy. Am J Respir Crit Care Med 2011, 184:514-520

4. Vincent JL, Roman A, Kahn RJ: Dobutamine administration in septic shock: addition to a standard protocol. Crit Care Med 1990, 18:689-693.

5. Schaffartzik W, Sanft C, Schaefer JH, Spies C: Different dosages of dobutamine in septic shock patients: determining oxygen consumption with a metabolic monitor integrated in a ventilator. Intensive Care Med 2000, 26:1740-1746.

6. Rivers EP, Katranii M, Jaehne KA, Brown S, Abou Dagher G, Cannon C, Coba V: Early interventions in severe sepsis and septic shock: a review of the evidence one decade later. Minerva Anestesiol 2012, 78:712-724.

7. Whippy A, Skeath M, Crawford B, Adams C, Marelich G, Alamshahi M, Borbon J: Kaiser Permanente's performance improvement system, part 3: multisite improvements in care for patients with sepsis. It Comm J Qual Patient Saf 2011, 37:483-493.

8. Cannon CM, Holthaus CV, Zubrow MT, Posa P, Gunaga S, Kella V, Elkin R Davis S, Turman B, Weingarten J, Milling TJ Jr, Lidsky N, Coba V, Suarez A, Yang JJ, Rivers EP: The GENESIS project (GENeralized Early Sepsis Intervention Strategies): a multicenter quality improvement collaborative. J Intensive Care Med 2013, 28:355-368.

9. Miller RR 3rd, Dong L, Nelson NC, Brown SM, Kuttler KG, Probst DR, Allen TL, Clemmer TP: Multicenter implementation of a severe sepsis and septic shock treatment bundle. Am J Respir Crit Care Med 2013, 188:77-82.
10. Puskarich MA, Marchick MR, Kline JA, Steuerwald MT, Jones AE: One year mortality of patients treated with an emergency department based early goal directed therapy protocol for severe sepsis and septic shock: a before and after study. Crit Care 2009, 13:R167.

11. Jones AE: Point: should lactate clearance be substituted for central venous oxygen saturation as goals of early severe sepsis and septic shock therapy? Yes Chest 2011, 140:1406-1408.

12. Hollenberg SM: Inotrope and vasopressor therapy of septic shock. Crit Care Clin 2009, 25:781-802. ix.

13. Wilkman E, Kaukonen KM, Pettila V, Kuitunen A, Varpula M: Association between inotrope treatment and 90-day mortality in patients with septic shock. Acta Anaesthesiol Scand 2013, 57:431-442.

14. Parsons EC, Hough CL, Seymour CW, Cooke CR, Rubenfeld GD, Watkins TR: Red blood cell transfusion and outcomes in patients with acute lung injury, sepsis and shock. Crit Care 2011, 15:R221.

15. Park DW, Chun BC, Kwon SS, Yoon YK, Choi WS, Sohn JW, Peck KR, Kim YS, Choi YH, Choi JY, Choi JY, Kim SI, Eom JS, Kim HY, Cheong HJ, Song YG, Choi HJ, Kim JM, Kim MJ: Red blood cell transfusions are associated with lower mortality in patients with severe sepsis and septic shock: a propensity-matched analysis*. Crit Care Med 2012, 40:3140-3145.

16. Marik PE, Sibbald WJ: Effect of stored-blood transfusion on oxygen delivery in patients with sepsis. JAMA 1993, 269:3024-3029.

17. Mazza BF, Machado FR, Mazza DD, Hassmann V: Evaluation of blood transfusion effects on mixed venous oxygen saturation and lactate levels in patients with SIRS/sepsis. Clinics (Sao Paulo) 2005, 60:311-316.

18. Sakr Y, Chierego M, Piagnerelli M, Verdant C, Dubois MJ, Koch M, Creteur J, Gullo A, Vincent JL, De Backer D: Microvascular response to red blood cell transfusion in patients with severe sepsis. Crit Care Med 2007, 35:1639-1644.

19. Hebert PC: Transfusion requirements in critical care (TRICC): a multicentre, randomized, controlled clinical study. Transfusion Requirements in Critical Care Investigators and the Canadian Critical care Trials Group. Br J Anaesth 1998, 81:25-33.

20. Villanueva C, Colomo A, Bosch A, Concepcion M, Hernandez-Gea V, Aracil C, Graupera I, Poca M, Alvarez-Urturi C, Gordillo J, Guarner-Argente C, Santalo M, Muniz E, Guarner C: Transfusion strategies for acute upper gastrointestinal bleeding. N Engl J Med 2013, 368:11-21.

21. Carson JL, Terrin ML, Noveck H, Sanders DW, Chaitman BR, Rhoads GG Nemo G, Dragert K, Beaupre L, Hildebrand K, Macaulay W, Lewis C, Cook DR, Dobbin G, Zakriya KJ, Apple FS, Horney RA, Magaziner J: Liberal or restrictive transfusion in high-risk patients after hip surgery. N Engl J Med 2011, 365:2453-2462.

22. Marik PE, Corwin HL: Efficacy of red blood cell transfusion in the critically ill: a systematic review of the literature. Crit Care Med 2008, 36:2667-2674.

23. Purdy FR, Tweeddale MG, Merrick PM: Association of mortality with age of blood transfused in septic ICU patients. Can J Anaesth 1997, 44:1256-1261.

24. Sakr Y, Lobo S, Knuepfer S, Esser E, Bauer M, Settmacher U, Barz D, Reinhart K: Anemia and blood transfusion in a surgical intensive care unit. Crit Care 2010, 14:R92.

25. Levy B, Nace L, Bollaert PE, Dousset B, Mallie JP, Larcan A: Comparison of systemic and regional effects of dobutamine and dopexamine in norepinephrine-treated septic shock. Intensive Care Med 1999, 25:942-948.

26. De Backer D, Creteur J, Dubois MJ, Sakr Y, Koch M, Verdant C, Vincent JL: The effects of dobutamine on microcirculatory alterations in patients with septic shock are independent of its systemic effects. Crit Care Med 2006, 34:403-408.

27. Hernandez G, Bruhn A, Luengo C, Regueira T, Kattan E, Fuentealba A, Florez J, Castro R, Aquevedo A, Pairumani R, McNab P, Ince C: Effects of dobutamine on systemic, regional and microcirculatory perfusion parameters in septic shock: a randomized, placebo-controlled, double-blind, crossover study. Intensive Care Med 2013, 39:1435-1443.

28. Enrico C, Kanoore Edul VS, Vazquez AR, Pein MC, de la Hoz RA P, Ince C, Dubin A: Systemic and microcirculatory effects of dobutamine in patients with septic shock. J Crit Care 2012, 27:630-638.

29. Georges H, Journaux C, Devos P, Alfandari S, Delannoy PY, Meybeck A, Chiche A, Boussekey N, Leroy O: Improvement in process of care and outcome in patients requiring intensive care unit admission for community acquired pneumonia. BMC Infect Dis 2013, 13:196.

30. Kumar A, Schupp E, Bunnell E, Ali A, Milcarek B, Parrillo JE: Cardiovascular response to dobutamine stress predicts outcome in severe sepsis and septic shock. Crit Care 2008, 12:R35. 
31. Jones AE, Shapiro NI, Trzeciak S, Arnold RC, Claremont HA, Kline JA: Lactate clearance vs central venous oxygen saturation as goals of early sepsis therapy: a randomized clinical trial. JAMA 2010, 303:739-746.

32. Nguyen HB, Kuan WS, Batech M, Shrikhande P, Mahadevan M, Li CH, Ray S, Dengel A: Outcome effectiveness of the severe sepsis resuscitation bundle with addition of lactate clearance as a bundle item: a multi-national evaluation. Crit Care 2011, 15:R229.

33. Arabi YM, Dara SI, Tamim HM, Rishu AH, Bouchama A, Khedr MK, Feinstein D, Parrillo JE, Wood KE, Keenan SP, Zanotti S, Martinka G, Kumar A, Kumar A: Clinical characteristics, sepsis interventions and outcomes in the obese patients with septic shock: an international multicenter cohort study. Crit Care 2013, 17:R72.

34. Jones AE, Focht A, Horton JM, Kline JA: Prospective external validation of the clinical effectiveness of an emergency department-based early goal-directed therapy protocol for severe sepsis and septic shock. Chest 2007, 132:425-432.

35. Nguyen HB, Corbett SW, Steele R, Banta J, Clark RT, Hayes SR, Edwards J, Cho TW, Wittlake WA: Implementation of a bundle of quality indicators for the early management of severe sepsis and septic shock is associated with decreased mortality. Crit Care Med 2007, 35:1105-1112

36. Escobar GJ, Greene JD, Scheirer P, Gardner MN, Draper D, Kipnis P. Risk-adjusting hospital inpatient mortality using automated inpatient outpatient, and laboratory databases. Med Care 2008, 46:232-239.

37. Berger T, Green J, Horeczko T, Hagar Y, Garg N, Suarez A, Panacek E, Shapiro N: Shock index and early recognition of sepsis in the emergency department: pilot study. West J Emerg Med 2013, 14:168-174

38. Nguyen HB, Rivers EP, Knoblich BP, Jacobsen G, Muzzin A, Ressler JA, Tomlanovich MC: Early lactate clearance is associated with improved outcome in severe sepsis and septic shock. Crit Care Med 2004, 32:1637-1642.

39. Kumar A, Haery C, Paladugu B, Kumar A, Symeoneides S, Taiberg L, Osman J, Trenholme G, Opal SM, Goldfarb R, Parrillo JE: The duration of hypotension before the initiation of antibiotic treatment is a critical determinant of survival in a murine model of Escherichia coli septic shock: association with serum lactate and inflammatory cytokine levels. J Infect Dis 2006, 193:251-258.

40. Namendys-Silva SA, Texcocano-Becerra J, Herrera-Gomez A: Application of the Sequential Organ Failure Assessment (SOFA) score to patients with cancer admitted to the intensive care unit. Am J Hosp Palliat Care 2009, 26:341-346

41. van Walraven $C$, Austin PC, Jennings $A$, Quan $H$, Forster AJ: A modification of the Elixhauser comorbidity measures into a point system for hospital death using administrative data. Med Care 2009, 47:626-633.

42. Liu V, Turk BJ, Ragins Al, Kipnis P, Escobar GJ: An electronic Simplified Acute Physiology Score-based risk adjustment score for critical illness in an integrated healthcare system. Crit Care Med 2013, 41:41-48.

43. Jones AE, Trzeciak S, Kline JA: The Sequential Organ Failure Assessment score for predicting outcome in patients with severe sepsis and evidence of hypoperfusion at the time of emergency department presentation. Crit Care Med 2009, 37:1649-1654.

44. Peduzzi $\mathrm{P}$, Concato J, Kemper $\mathrm{E}$, Holford TR, Feinstein AR: A simulation study of the number of events per variable in logistic regression analysis. J Clin Epidemiol 1996, 49:1373-1379.

45. Rosenbaum PR, Rubin DB: Reducing bias in observational studies using subclassification on the propensity score. J Am Stat Assoc 1984, 79:516-524.

46. Vittinghoff $E$, McCulloch CE: Relaxing the rule of ten events per variable in logistic and Cox regression. Am J Epidemiol 2007, 165:710-718.

47. Yealy DM, Kellum JA, Huang DT, Barnato AE, Weissfeld LA, Pike F, Terndrup T, Wang HE, Hou PC, LoVecchio F, Filbin MR, Shapiro NI, Angus DC: A randomized trial of protocol-based care for early septic shock. N Engl J Med 2014, 370:1683-1693.

48. Vincent JL, Sakr Y, Sprung C, Harboe S, Damas P: Are blood transfusions associated with greater mortality rates? Results of the Sepsis Occurrence in Acutely III Patients study. Anesthesiology 2008, 108:31-39.

49. Sparrow RL: Red blood cell storage and transfusion-related immunomodulation. Blood Transfus 2010, 8:s26-s30.

50. Sadaka F, Aggu-Sher R, Krause K, O'Brien J, Armbrecht ES, Taylor RW: The effect of red blood cell transfusion on tissue oxygenation and microcirculation in severe septic patients. Ann Intensive Care 2011, 1:46.
51. Locke R, Paul D, Touch S, Mackley A, Maduskuie V, Fawcett P: Cytokine load in prestorage leukoreduced PRBC transfusions in premature infants. J Perinatol 2005, 25:526-530.

52. Hartemink KJ, Groeneveld AB: Vasopressors and inotropes in the treatment of human septic shock: effect on innate immunity? Inflammation 2012, 35:206-213.

53. Kern H, Schroder T, Kaulfuss M, Martin M, Kox WJ, Spies CD: Enoximone in contrast to dobutamine improves hepatosplanchnic function in fluid-optimized septic shock patients. Crit Care Med 2001, 29:1519-1525.

54. Morelli A, Ertmer C, Westphal M, Rehberg S, Kampmeier T, Ligges S, Orecchioni A, D'Egidio A, D'Ippoliti F, Raffone C, Venditti M, Guarracino F, Girardis M, Tritapepe L, Pietropaoli P, Mebazaa A, Singer M: Effect of heart rate control with esmolol on hemodynamic and clinical outcomes in patients with septic shock: a randomized clinical trial. JAMA 2013, 310:1683-1691.

55. Nallamothu BK, Hayward RA, Bates ER: Beyond the randomized clinical trial: the role of effectiveness studies in evaluating cardiovascular therapies. Circulation 2008, 118:1294-1303.

56. Casserly B, Gerlach H, Phillips GS, Lemeshow S, Marshall JC, Osborn TM, Levy MM: Low-dose steroids in adult septic shock: results of the Surviving Sepsis Campaign. Intensive Care Med 2012, 38:1946-1954.

57. Sprung $C L$, Annane $D$, Keh $D$, Moreno $R$, Singer M, Freivogel $K$, Weiss $Y G$, Benbenishty J, Kalenka A, Forst H, Laterre PF, Reinhart K, Cuthbertson BH, Payen D, Briegel J: Hydrocortisone therapy for patients with septic shock. N Engl J Med 2008, 358:111-124.

58. Annane D, Sebille V, Charpentier C, Bollaert PE, Francois B, Korach JM, Capellier G, Cohen Y, Azoulay E, Troche G, Chaumet-Riffaud P, Bellissant E: Effect of treatment with low doses of hydrocortisone and fludrocortisone on mortality in patients with septic shock. JAMA 2002, 288:862-871.

59. Annane D, Briegel J, Keh D, Moreno R, Singer M, Sprung CL: Clinical equipoise remains for issues of adrenocorticotropic hormone administration, cortisol testing, and therapeutic use of hydrocortisone. Crit Care Med 2003, 31:2250-2251. author reply 2252-2253.

\section{doi:10.1186/s13054-014-0496-y}

Cite this article as: Mark et al:: In-hospital mortality following treatment with red blood cell transfusion or inotropic therapy during early goal-directed therapy for septic shock: a retrospective propensity-adjusted analysis. Critical Care 2014 18:496.

\section{Submit your next manuscript to BioMed Central and take full advantage of:}

- Convenient online submission

- Thorough peer review

- No space constraints or color figure charges

- Immediate publication on acceptance

- Inclusion in PubMed, CAS, Scopus and Google Scholar

- Research which is freely available for redistribution 